\title{
Influence of Mirrors utilization on the Radiation Emitted by Models Subjected to Hypersonic Flow for Surface Temperature Determination
}

\author{
by F. De Filippis*, C. Toscano**, D. Gallo***, P. Caruso***and L.Savino*****
}

${ }^{*}$ C.I.R.A. - Italian Aerospace Research Centre, via Maiorise sn, 81043 Capua (CE) Italy, f.defilippis@cira.it

${ }^{*}$ C.I.R.A. - Italian Aerospace Research Centre, via Maiorise sn, 81043 Capua (CE) Italy, c.toscano@cira.it $\mathrm{PhD}$ at DIAS

*** Second University of Naples, Information Engineering Department, Via Roma 29, Aversa (CE), daniele.gallo@unina2.it

**** Second University of Naples, Information Engineering Department, Via Roma 29, Aversa (CE), carusopasquale1985@yahoo.it

${ }^{\star * * * *}$ C.I.R.A. - Italian Aerospace Research Centre, via Maiorise sn, 81043 Capua (CE) Italy, I.savino@cira.it

This work focuses on the specific application of Infrared Thermography (IRT) during plasma wind tunnel tests that reproduce hypersonic gas conditions performed in CIRA Scirocco facility. Unfortunately some drawbacks can limit the use of the thermographic set up in Scirocco in some specific nozzle configurations, because it is impossible to obtain a direct view of the test model from the thermal camera. These difficulties are overcome by using a mirror, which, on the contrary, provides a non direct view of the model by the thermal camera. The present work shows that classical mirrors (with reflectivity across the entire visible spectrum) can represent a simple and cheap way to measure the target temperature in these critical geometric conditions.

\section{Introduction}

IR thermography is a very useful technique for temperature measurement over model surfaces tested in Plasma Wind Tunnels (PWT) without any physical contact with the object under analysis.

The signal, in this case goes through different means before impinging the thermal sensor: plasma, lens systems, filter etc. The result is a two-dimensional map of temperature of the analyzed object. This technique is very useful mainly for three reasons:

- the possibility to make a measurement at a certain distance without any contact with the object (there is no body modification as necessary for the thermocouples);

- $\quad$ its capability to provide a two dimensional map in which, the surface temperatures of the framed objects are shown, then it can be very quick and easy to make a comparison between different areas of the body and to detect the critical points;

- $\quad$ it provides real time visualization of the analyzed phenomenon.

In this work thermography has been used for temperature determination over model surfaces subjected to the hypersonic flow generated by Scirocco facility. In order to visualize the model from a lateral side, a mirror has been used since some Plasma Wind Tunnel conical nozzle configurations didn't allow to directly frame the model. This work represents and discusses the results obtained during three recently performed test campaigns, named in the following 1)MHD, 2)AURORA and 3) RASTAS.

\section{Facility and thermographic system features}

The SCIROCCO Plasma Wind Tunnel is an arc heated facility, capable of producing a low-pressure high-enthalpy hypersonic flow of large dimension impinging on samples in scales up to 1:1 with a test duration up to 30 minutes. Until now the facility's most important employ concerns with testing and validating Thermal Protection System materials. The design and engineering of the SCIROCCO PWT represent the state of art for the arc jet technology. A facility schema is reported in fig. 1 [1].

In the facility Test Chamber air is accelerated through a convergent-divergent conical nozzle. To reach hypersonic speed. There are five different nozzle configurations in order to achieve the desired flow condition and to match any model size (tab.1) [2], [3].A Model Support System inserts the test article in the plasma flow.

\begin{tabular}{|c|c|c|c|c|c|}
\hline Conical Nozzle & $\mathbf{0}$ & c & d & e & f \\
\hline Diameter & $187 \mathrm{~mm}$ & $900 \mathrm{~mm}$ & $1150 \mathrm{~mm}$ & $1350 \mathrm{~mm}$ & $1950 \mathrm{~mm}$ \\
\hline
\end{tabular}

Table 1. Diameters of different conical nozzle configuration 
In the test campaigns two cameras were used: Agema Thermovision 900 and Flir SC 5500. They were located near to a Test Chamber lateral window and to a top side window as you can see in fig. 1 .

In the case of the lateral window location, a mirror placed in the test chamber, was properly oriented in order to allow the Agema to frame the sample to be tested.
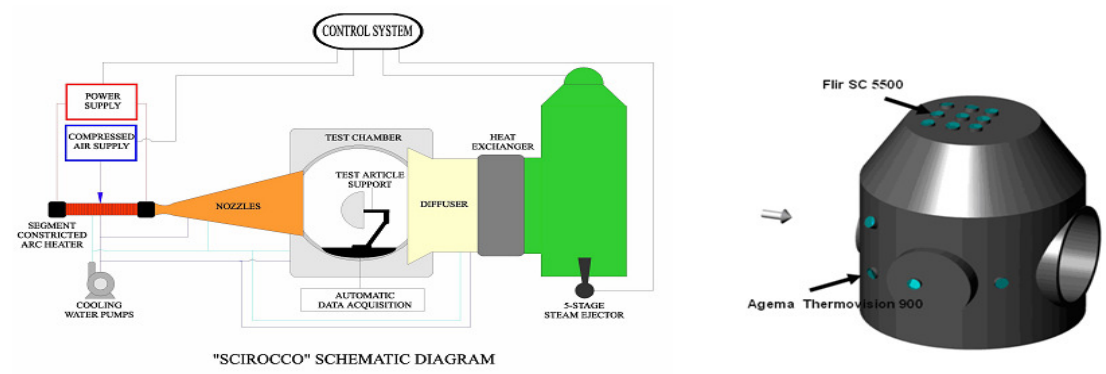

Fig.1. Facility schema and scheme of the wndows used for the cameras

$i$

The Agema Thermovision 900 acquisition system works in a spectral range of $8 \div 12 \mu \mathrm{m}$ and can perform measures with or without filters. Maximum detectable temperature value for no filter conditions is about $1500^{\circ} \mathrm{C}$; this threshold can be extended up to $3000^{\circ} \mathrm{C}$ through the use of the three filters available. To increase the accuracy (relative to $1 \%$ FS) any filter permits also acquisition range in more smaller ranges. The tab. 2 shows the ranges associated to the different filters of the camera.

\begin{tabular}{|c|c|c|c|c|c|c|c|}
\hline & Range & & Range & & Range & & Range \\
\hline \multirow{4}{*}{ No filter } & $-30 \div 80^{\circ} \mathrm{C}$ & \multirow{4}{*}{ LPL } & $0 \div 110^{\circ} \mathrm{C}$ & \multirow{4}{*}{ X42 } & $0 \div 350^{\circ} \mathrm{C}$ & \multirow{4}{*}{ X59 } & $100 \div 350^{\circ} \mathrm{C}$ \\
\hline & $0 \div 250^{\circ} \mathrm{C}$ & & $50 \div 350^{\circ} \mathrm{C}$ & & $100 \div 1200^{\circ} \mathrm{C}$ & & $150 \div 500^{\circ} \mathrm{C}$ \\
\hline & $50 \div 600^{\circ} \mathrm{C}$ & & $150 \div 1000^{\circ} \mathrm{C}$ & & $250 \div 3000^{\circ} \mathrm{C}$ & & $250 \div 850^{\circ} \mathrm{C}$ \\
\hline & $250 \div 1500^{\circ} \mathrm{C}$ & & $250 \div 2000^{\circ} \mathrm{C}$ & & & & $500 \div 2000^{\circ} \mathrm{C}$ \\
\hline
\end{tabular}

Table 2. Operative ranges of Thermovision Agema 900

The SC5500 acquisition system works connected to a computer through Ethernet cable. His spectral response curve acts in the range $(3.7 \div 5) \mu \mathrm{m}$ and the accuracy is $1 \%$ of the reading value.

During SCIROCCO tests it is necessary to locate the cameras at the external part of the facility because of the vacuum conditions inside the test chamber. For this reason the measurements are performed through a window of Zincum and Selenium ( $\mathrm{ZnSe}$ ) with a special coating that guarantees a passing band in the Agema working range $(8 \div 12) \mu \mathrm{m}$.

During the MHD test campaign, LPL filter mounted on Agema Thermovision 900, for range $(150 \div 1000){ }^{\circ} \mathrm{C}$, was used, while no filter condition and range $(250 \div 1500){ }^{\circ} \mathrm{C}$ were used in AURORA and RASTAS test campaigns.

The SC5500 camera works in a different spectral range with respect to the previous one, then the optical window, in this range, acts as an attenuation filter. Moreover the thermal camera is provided already calibrated in a smaller temperature range $(-10 \div 300)^{\circ} \mathrm{C}$ than the required one for the tests. In order to enhance the thermal camera performance, an appropriate attenuation filter has been mounted. It is a sapphire narrow band filter provided by FLIR, with Central Wavelength (CW) of $3,99 \mu \mathrm{m}$ and a transmission peak larger than $60 \%$.

In order to obtain correct measures of the temperature by the two thermal cameras, both of them were subjected to a calibration by using specific Black Bodies (BB) available in CIRA laboratories

\section{Calibration: theoretical principles}

The calibration is necessary to know the correspondence between the radiation impinging onto the detector sensor and the provided temperature.

Since the temperature measurement can, generally, be affected by atmospheric damping, and radiations reflected by the surrounding objects the radiation $\left(S^{\prime}\right)$ on the detector will be :

$$
S^{I}=\tau \cdot \varepsilon \cdot S_{0}+\tau \cdot(1-\varepsilon) \cdot S_{a}+(1-\tau) \cdot S_{a t m}
$$


where $S_{o}$ is the emitted radiation by the $\mathrm{BB}$ at the same temperature than the body analyzed, $\mathrm{S}_{\mathrm{a}}$ is the radiation emitted by the environment (this term concerns with reflections). and $S_{a t m}$ is the radiation emitted by atmosphere (this contribution, in our specific case, can be neglected since the distance between the thermal camera and the bottom of the black body cavity is short)[4].

The thermography systems provide a number called Object Signal, in the case of the AGEMA 900 (OS or I) and a number called Digital Level in the case of the SC5500 (DL or I); both are proportional to $S^{\prime}$ and with a significance similar to the relationship (1), it is possible to write that

$$
I=\tau \cdot \varepsilon \cdot I_{0}+\tau \cdot(1-\varepsilon) \cdot I_{a}+(1-\tau) \cdot I_{a t m}
$$

Measured temperature is function of OS and of the emissivity of the body analyzed. Calibration curve comes from an iterative regression technique, using this equation:

$$
I=\frac{R}{e^{\frac{B}{T}}-F}
$$

where:I is Thermal Value (Object Signal), $\mathrm{R}$ is the Response factor, B is the Spectral Factor,F is the Shape Factor and T is the temperature of the object $(\mathrm{K})$.

Camera calibration concerns with the determination of the three parameters $(R, B, F)$ via a data fitting: in that way, the output given by the camera will be as close as possible to the real temperature of the BB.

It is important to underline that, generally, the calibration has to be performed because of a possible loss of sensitivity of the detector after years of camera using.

Inverting the (3) an expression of the temperature as function as the object signal can be obtained.

$$
T=\frac{B}{\ln \left(F+\frac{R}{I}\right)}
$$

In this way, the obtained calibration curve provides a more accurate correspondence between the gray levels and the temperature revealed by the infrared camera with respect to the temperature obtained before the calibration; moreover, this procedure doesn't introduce any change in the acquisition system specifications, in terms of accuracy of the measurement.

For the calibration of both cameras in the specific used range, the black body (BB) Mikron M305 and M390 were used. Black body (BB) M305 can reach temperatures in a range that goes from $300^{\circ} \mathrm{C}$ to $1000^{\circ} \mathrm{C}$., whereas $\mathrm{BB}$ M390 can reach temperatures in a range that goes from $600{ }^{\circ} \mathrm{C}$ to $3000{ }^{\circ} \mathrm{C}$.

\section{Test analyzed}

In this work thermographic results are described for three test campaigns performed in Scirocco.

In particular, MHD test campaign consisted in the study of the influence of a magnetic field generated by an electromagnet inserted into the model on its thermal and mechanical hypersonic loads. For this test the conical nozzle $F$ configuration has been used [5].

In AURORA and RASTAS test campaign, the 0 configuration conical nozzle has been used to simulate earth re-entry from interplanetary trajectories. In Aurora the models were realized in amorphous carbon, while in RASTAS test campaign, a $75 \mathrm{~mm}$ diameter hemispherical body with ceramic ablative material over the surface has been tested through 0 configuration conical nozzle [6], [7].

For these test campaigns thermography played a fundamental role in the investigation of temperature distribution over the model surface and hence for analysis of the tested materials and technologies, since it allowed to reveal the absolute temperature over the model surface together with its gradients [8]. 

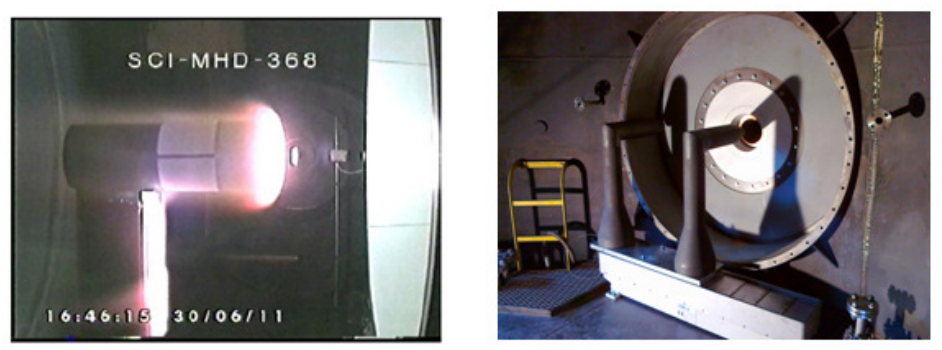

Fig.2. Image of MHD test in Scirocco and of the configuration used for AURORA and RASTAS test campaigns

\section{Agema Thermovision 900 and SC 5500 Calibration}

Fig. 3 refers to the set up used for calibration in MHD test campaign. During the test it was not possible to frame the overall model (as you can see in Fig. 3), locating the camera in correspondence of a lateral window because the $F$ configuration for the divergent nozzle (with a diameter of about 2 meters) was used. This nozzle reduced the field of view from a lateral side, so the use of a mirror was necessary to view the model and to capture the entire transversal surface (in order to capture the stagnation point). The calibration has been in this way a necessity because the mirror significantly affected the radiation attenuating it.

The inclination of the mirror was such as to capture the whole image of model that could be seen so through the camera. In Tab. 3 the features of the mirror used are showed:

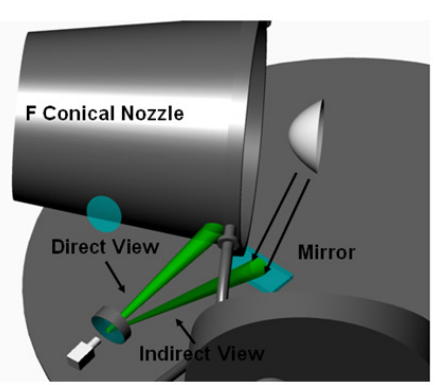

Fig.3. 3d scheme representative of the perspective of the lateral camera used in MHD test

\begin{tabular}{|l|l|}
\hline Dimensions $(\mathrm{mm})$ & $408 \times 609$ \\
\hline Thickness $(\mathrm{mm})$ & 6.00 \\
\hline Surface Accuracy $(\lambda)$ & $4-6$ per Inch \\
\hline Substrate & Soda Lime Float Glass \\
\hline Coating & $\begin{array}{l}\text { Enhanced Aluminum, R }>90 \% \text { from } 400- \\
650 \mathrm{~nm}\end{array}$ \\
\hline
\end{tabular}

Table 3. Mirror features

In Tab. 4 the R, B, and F parameters without the mirror and the same parameters calculated after the calibration are reported and the significant difference can be observed.

\begin{tabular}{|c|r|r|r|}
\hline $\begin{array}{c}\text { Old parameters } \\
\text { (pefore } \\
\text { calibration) }\end{array}$ & \multicolumn{2}{|c|}{$\begin{array}{c}\text { New Parameters } \\
\text { (after calibration) }\end{array}$} \\
\hline $\mathrm{R}=$ & $3637 \mathrm{R}$ & $\mathrm{R}=$ & 80,73 \\
\hline $\mathrm{B}=$ & $1329, \mathrm{8}$ & $\mathrm{B}=$ & 60,25 \\
\hline $\mathrm{F}=$ & 1,1 & $\mathrm{~F}=$ & 1,05 \\
\hline
\end{tabular}

Table 4. Comparison between Agema $R, B$ and $F$ parameters before and after calibration for its use in MHD test

In the calibration procedure for MHD test, the Mikron M310 black body temperature has been set starting from $100{ }^{\circ} \mathrm{C}$ and reaching the maximum value of $350{ }^{\circ} \mathrm{C}$ with step of $25^{\circ} \mathrm{C}$.

To analyse the mirror effects fig. 4 has to be analysed. For temperature of the same size order of those obtained in the MHD test campaign (about $160^{\circ} \mathrm{C}$ ), it is observed a $65 \%$ of percentage difference between the temperature measured and the effective one generated through the black body considered as reference. Neglecting the term due to the absorption of radiation by the air, whose trasmissivity can be considered as 1 , and the reflection of the environment 
(emissivity is in fact imposed as 1), the attenuation in temperature, as consequence, is mainly due to the mirror that is characterized by an high reflectivity in the visible range but an high absorption of the radiation in infrared range.

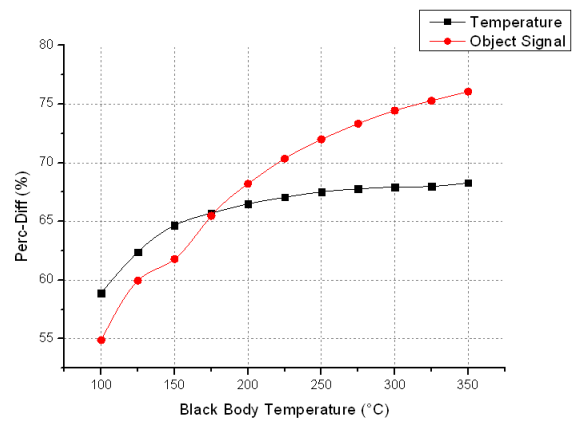

Fig.4. Percentage difference between temperature measured by Agema camera (with mirror) and BB temperature and between the OS associated to temperature measured and that associated to BB temperature

During the AURORA and RASTAS test campaigns in SCIROCCO, the "0" configuration for the divergent nozzle (with a diameter of about $18 \mathrm{~cm}$ ) was used and the thermal camera was placed in correspondence of a lateral window of the Test Chamber. Due to this configuration and to the used nozzle, which seriously reduced the field of view of the camera, the use of a mirror was needed to frame the model (fig.5). The mirror main features are reported in tab.3. In tab. 5 the R, $\mathrm{B}$, and $\mathrm{F}$ parameters, without mirror, and the same parameters calculated after the calibration are shown.

\begin{tabular}{|c|r|r|r|}
\hline \multicolumn{2}{|c|}{$\begin{array}{c}\text { Old parameters } \\
\text { (before calibration) }\end{array}$} & \multicolumn{2}{|c|}{$\begin{array}{c}\text { New Parameters (after } \\
\text { calibration) }\end{array}$} \\
\hline $\mathrm{R}=$ & 10948 & $\mathrm{R}=$ & 1439,54 \\
\hline $\mathrm{B}=$ & 1522,3 & $\mathrm{~B}=$ & 1206,34 \\
\hline $\mathrm{F}=$ & 1,2 & $\mathrm{~F}=$ & 1,06 \\
\hline
\end{tabular}

Table 5. Comparison between Agema R, B and F parameters before and after calibration for its use in Aurora and RASTAS tests

In the calibration procedure for RASTAS test, the starting temperature of the Mikron M390 black body was set at almost $600{ }^{\circ} \mathrm{C}$, then it was increased up to the maximum value of $2400{ }^{\circ} \mathrm{C}$. The fig. 5 reports the same percentage difference representation as in the previous fig. 4 and shows that the signal is severely attenuated by the mirror also at temperatures reached in AURORA and RASTAS test campaign (about $1700^{\circ} \mathrm{C}$ )
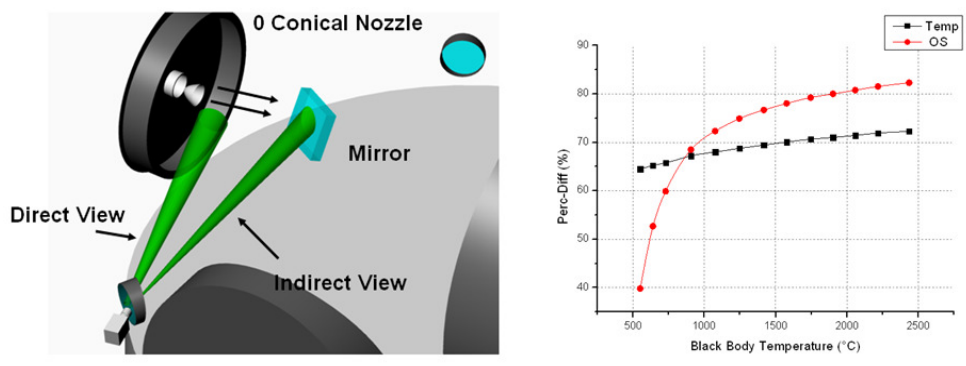

Fig.5. Mirror position 3d representation (on the left) and percentage difference between temperature measured by Agema camera and BB temperature revealed during calibration for Aurora and Rastas test campaigns (and between the OS associated to temperature measured and that associated to BB temperature) (on the right)

Flir SC 5500 camera framed the model from a top window (fig.1). For this kind of camera an attenuation effect has been mainly due to the optical window used described in the previous paragraph. The procedure for the calibration of Flir SC 5500 has been analogous to that use for the Agema 900, interposing in thelaboratory the specific window between the $\mathrm{BB}$ and the camera 


\section{MHD test results}

MHD test has been performed in two days; in the first day the electromagnet ignition was activated two times at a lower inlet facility power, while in the second day it was activated three times at higer power. Only the first test results are presented in this work.

The calibrated thermography results represented the more important information used for the data elaboration. At first the instant at which the model was carried at centreline TCL has been detected, then the time ranges of the electromagnet ignitions were extracted. In the next images (Fig. 6-7) the spatial trend has been reported for two perpendicular lines on the frontal surface of the model. For any ignition three instants were considered, i.e. 5 seconds before the ignition, the central instant during the ignition, and 5 seconds after the ignition. In this way it was possible to deduce the temperature values on the transversal surface and at the same time to make an investigation of the possible electromagnetic influence on the temperature trend.

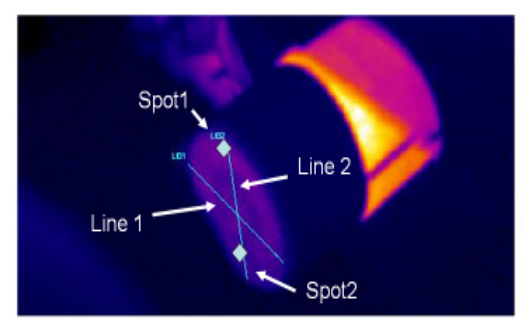

Fig.6. Analysed lines and spots positioning on the model used in MHD test

The first magnet ignition was activated 60 seconds after the model positioning along the center line, while the second one has been activated two minutes after the achievement of the center line condition. The graphs were elaborated by setting an emissivity of 0.7. This value came from a comparison between the two cameras (Agema 900 and SC 5000) and it was fixed because it gave compatible values of temperature for the two cameras at specific spots analyzed. Temperature trends along the described lines for the two ignitions are reported below.
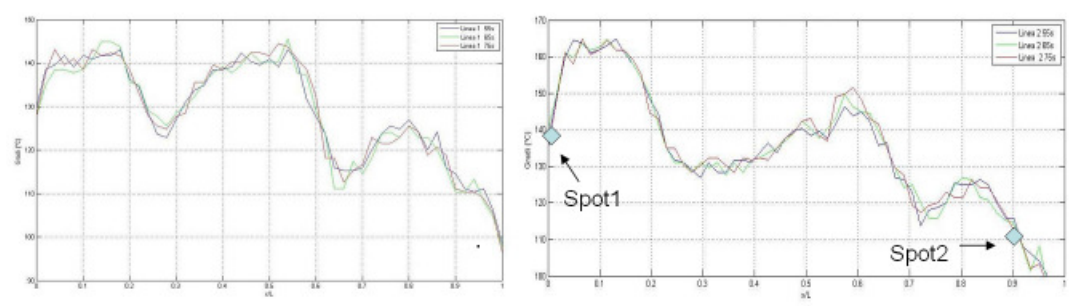

Fig.7. Temperature trend along the line1 (left), line2 (right), for the three main temporal instants ( $11=55$ s before the electromagnet ignition, $t 2=65$ s during the magnet working, $t 3=75$ s after the magnet power off (data relevant to the first ignition first ignition)

Temporal trend of temperature and pressure for spots placed on the frontal surface are reported in fig. 8
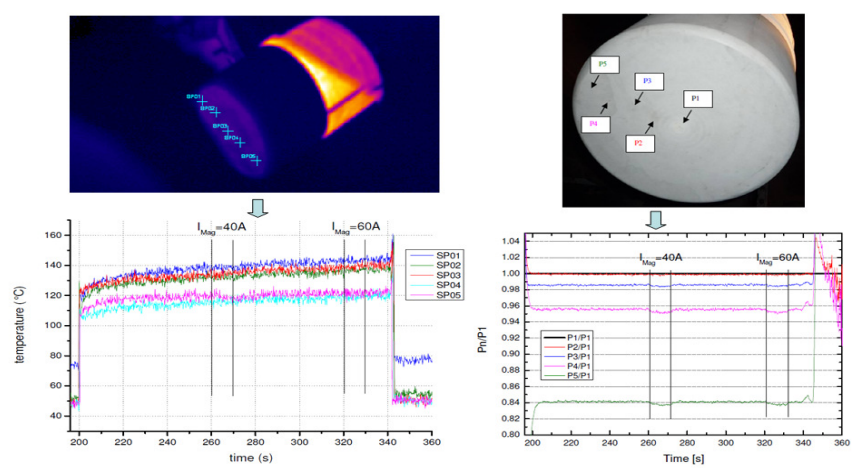

Fig.8. Surface temperature (left) and static pressure (right) temporal trend during MHD test 
As you can see in fig. 8 temperature trend shows fluctuations due to the effect of the mirror of about $5{ }^{\circ} \mathrm{C}$. For the two electromagnet ignitions, a variation in pressure of about $1 \%$ of the read value can be noticed in fig. 8 on the right, while variation of temperature was not revealed.

For Flir SC 5500 camera the temperature was determined along the line represented in fig. 9.
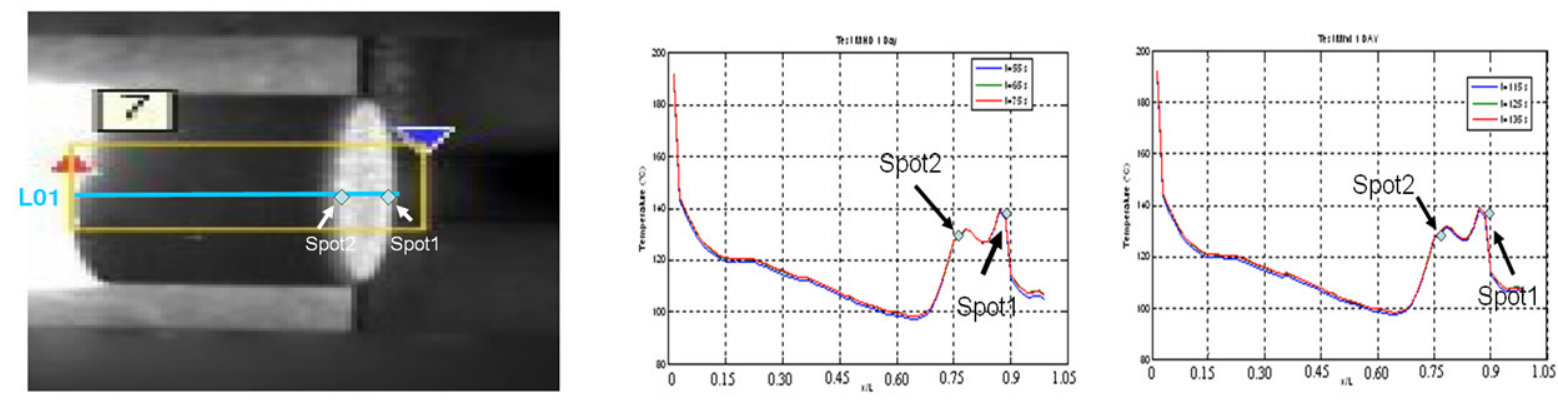

Fig.9. Image of the model as seen by the Flir SC 5500 (first on left) camera and temperature trend along the line for the three main temporal instants (first ignition on the second image, second ignition on the third image)

Previous figures show agreement between the temperatures measured with two cameras especially if we consider the 2 spots (spot 1 and 2) as reference for comparison between the two cameras.

\section{Aurora test results}

AURORA test has been performed with a "0" configuration nozzle. In this test campaign a $75 \mathrm{~mm}$ diameter hemispherical body has been tested. During the test, and the consequent temperature evolution acquisition, the emissivity was set at 0.8. In the following fig. 10, the maximum thermal stress condition of the model during the test, from a lateral point of view, is thermographically represented. The image represents the model taken just a frame before the model breaking instant. It has been exposed at an high enthalpy flow for about 8 seconds until it was subjected to breaking point.
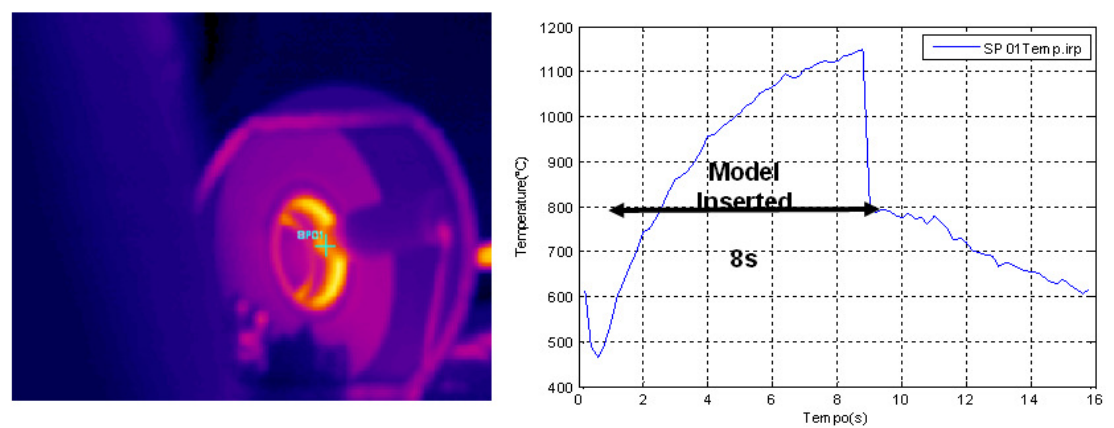

Fig.10. Spot analyzed over the model in Aurora test campaign

The emissivity was set at 0.8 also for the SC 5500 thermal camera. The frame frequency was $5 \mathrm{~Hz}$.

In the following fig. 11, the model facing the chamber throat is thermographically represented, moreover the position of the pointer used to monitor the temperature evolution is shown. The image is taken just only a frame before the model breaking point is reached. 

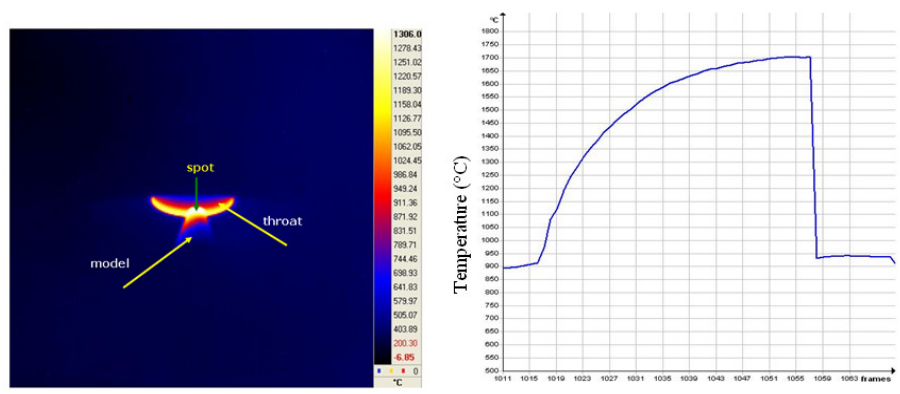

Frames

Fig.11. The model head facing the throat is shown (left) and temperature evolution of the model head during the test (right) (Aurora test)

The head of the model reached a maximum temperature of circa $1700{ }^{\circ} \mathrm{C}$ until its breaking. In the fig. 11 (right), the temperature evolution of the head pointer is plotted. In the figure, the maximum temperature achieved can be easily appreciated as well as the temperature abrupt decreasing after the model breaking.

Due to the different position of the used thermal cameras, it is not possible to overlap the images provided by each of them. In fact, the SC5500 has a view from above of the model during the test, with a view of a region closer to the stagnation point, whereas the AGEMA provide a lateral view through the reflection in the mirror, which did not allow to get images coming from the head of the model. Anyway, due to the axial-simmetry of the model, it is possible to identify, in the images provided by []$_{1}$ د $: \leqslant: 500$ (fig. 12, first image from left) a point belonging to an area which can be considered as an isotherm (in terms of the thermal evolution during the test) which behaves similarly to the inspected point provided by the AGEMA (fig. 12 second image from left).

This can be easily appreciated in fig. 12 (third image from left), where the temperature-time graphs for the two spots are plotted.
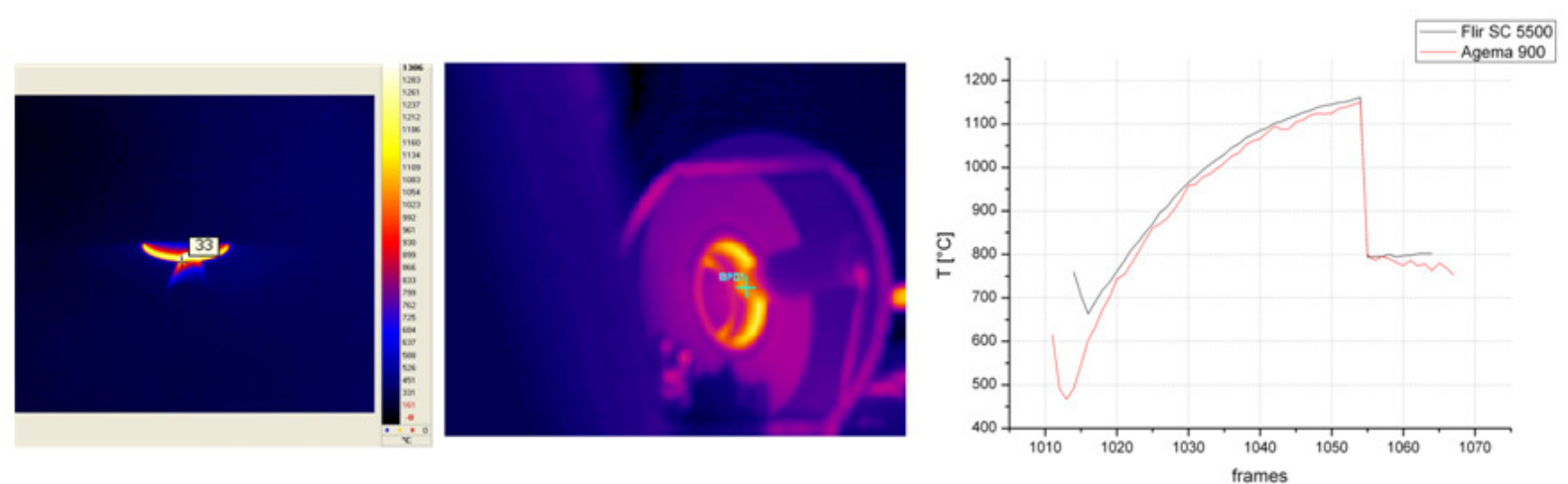

Fig.12. Spot "33" on an isothermal area of the model, i.e. an area in which all the points on the model have similar temperature-time behaviour (Aurora test) and comparison with an equivalent spot taken in the Agema frame

\section{Rastas test results}

Rastas test was performed with a "0" configuration nozzle. In this test campaign a $75 \mathrm{~mm}$ diameter hemispherical body has been and during the test, the emissivity was set at 0.8 . In the following fig. 13, the maximum thermal stress condition during the test, from a lateral point of view, is thermographically represented. The model has been exposed at an high enthalpy flow for about 13 seconds before it was subjected to breaking point. 

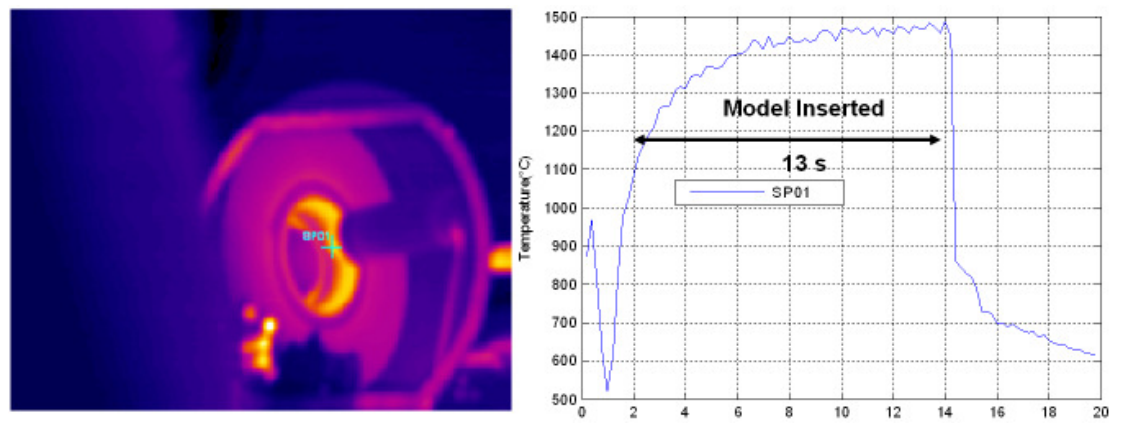

Fig.13. Spot analyzed over the model in Rastas test campaign

The emissivity was set at 0.8 also for SC 5500 thermal camera. The frame frequency was $15 \mathrm{~Hz}$.

In the following fig. 14 the model facing the chamber throat is shown, moreover the pointer used to obtain temperature evolution is shown. The image is taken just a frame before the model breaking point is reached.
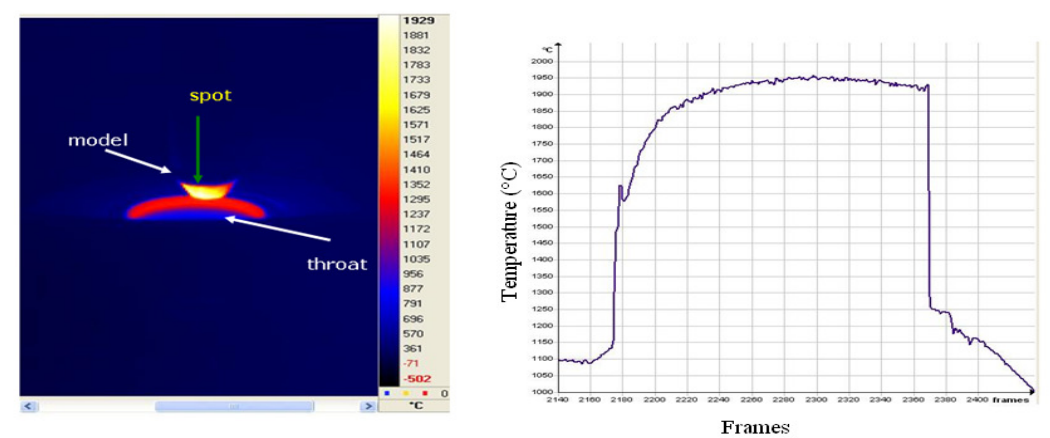

Fig.14. The model head facing the throat is shown (left) and temperature evolution of the model head during the test (right) (Rastas test)

The head of the model reached a maximum temperature of circa $1950{ }^{\circ} \mathrm{C}$ until its breaking. In the figure, the maximum temperature achieved can be easily appreciated as well as the temperature abrupt decreasing after the model breaking. Also for this test a comparison between the two camera data has been possible, following the same method described for the AURORA case, this can be appreciated in fig. 15, where the temperature-time graphs for the two spots are plotted.
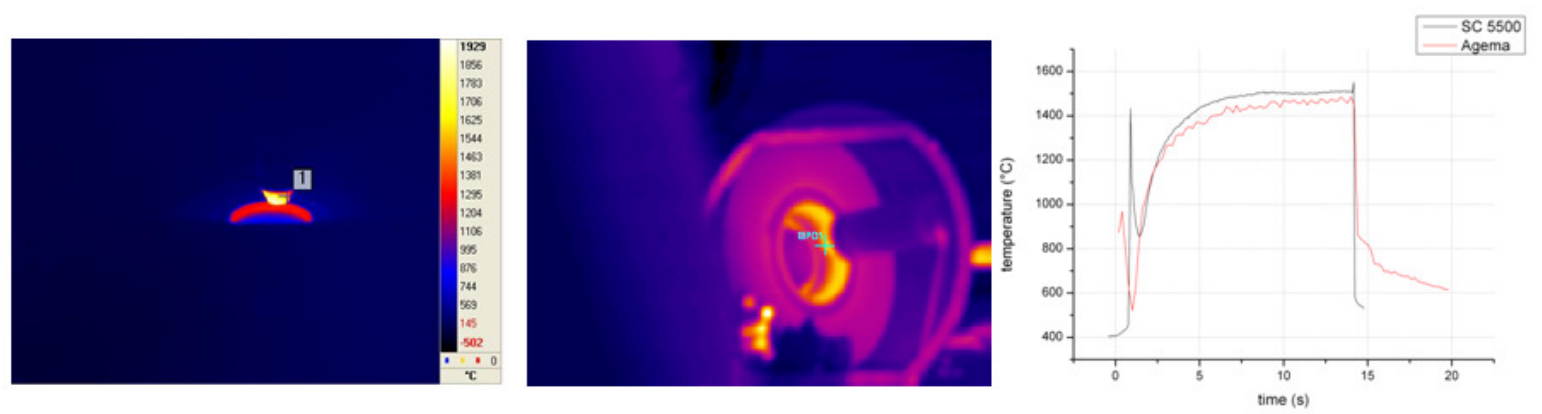

Fig.15. Spot "1" on an isothermal area of the model, i.e. an area in which all the points on the model have similar temperature-time behaviour (Rastas test) and comparison with an equivalent spot taken in the Agema frame

In fig.15 (third image from left) as you can notice, the heating phase in red curve (Agema camera) starts some frame (corresponding at about $0.5 \mathrm{~s}$ in time) before the black one (Flir camera). This is due to the different perspective by which the two cameras look at the model. In fact the spot analyzed by the side camera is occupied by the support when the model enters into the flow 
before it reaches the centerline. For Flir SC5500, in fig. 15 it is possible to appreciate firstly a fast increase of temperature and a subsequent slower increasing. This first peak was located at about 3 seconds. It is probably due to the fact that in the starting phase of the insertion, the camera sees an hotter point over the model at the analysed spot.

\section{Conclusions}

In the presented work a simple way for making indirect thermography measurements for evaluation of temperature distribution over surfaces of models subjected to hypersonic flows has been showed. A simple soda lime float glass mirrors with aluminium coating has been used, reflective in visible range. The accurate calibration to take into accounts the attenuation has been realised in laboratory using specific black bodies. The possibility to put the mirror in an environment characterized by low pressure condition (about $0.1 \mathrm{mbar}$ ) and with flow recirculation at the external side of the plasma has been demonstrated

Three test campaigns have been analyzed, named MHD, AURORA and RASTAS,

In MHD campaign thermography allowed to obtain two-dimensional maps of temperature over the model and to verify possible temperature changes due to electromagnetic generated fields. An attenuation of about $70 \%$ in the Object Signal has been generated by the mirror in the analysed range $100-350^{\circ} \mathrm{C}$. The AURORA test has been performed to simulate re-entry from interplanetary trajectories (such as those typically used in moon and mars missions) and the heat fluxes and loads deriving from this kind of space missions.

During the test, the model has been framed by two thermal cameras previously calibrated. A good agreement between the temperature-time graphs of two points taken on the model, from two cameras, with and without mirror respectively has been observed. In this case the calibration phase highlighted an attenuation generated by the mirror of about $80 \%$ in the Object Signal.

The RASTAS test campaigns confirmed the capabilities and possibilities to use mirrors, showing results qualities similar to that obtained during the AURORA test campaigns.

Finally those test campaigns show how, though the strong attenuation generated by the mirror, this simple experimental way to measure the temperature in hypersonic conditions gives good results and no particular problem induced by the environment where mirror was put (test chamber where low pressure and recirculation at the external part of plasma can occur) ,except some oscillations of about $5{ }^{\circ} \mathrm{C}$, were revelead in temperature temporal trend (this oscillations infact relative to the absolute values of temperature for the described tests, wherein any case usually temperature higher than $100{ }^{\circ} \mathrm{C}$ occur can be neglected).

\section{REFERENCES}

[1] F. De Filippis, A. Del Vecchio, S. Caristia, E. Graps SCIROCCO arc-jet facility for large scale spacecraft TPS verification Proceedings of 55th International Astronautical Congress, 4-8 October 2004, Vancouver (Canada).

[2] Russo G.,De Filippis F., Borrelli S., Marini M., Caristia S., "CIROCCO 70-MW Plasma Wind Tunnel: A New Hypersonic Capability" contribution to the AIAA 'Advanced Hypersonic Test Facilities' book, Chapter 11, pp. 313-351, 2002.

[3] De Filippis F., Caristia S., Del Vecchio A., Purpura C., "The SCIROCCO PWT Facility calibration activities" Proceedings of the 3rd International Symposium on Atmospheric Re-entry Vehicles and Systems, Arcachon (France), 24-27 March, 2003.

[4] Maldague X. P. V., "Theory and Practice of Infrafred Technology for Nondestructive Testing". John Wiley \& Sons, Inc., New York, 2001.

[5] A.Cristofolini, C.A. Borghi, G.Neretti, F.De Filippis, L.Savino, E.Marenna, "Experimental Investigations On The Hypersonic Nitrogen Flow For Magneto Fluid Dynamic Interaction Around a Blunt Body", 41st AIAA Fluid Dynamics Conference, Honolulu, June 27-30, 2011.

[6] Marino G., D Filippis F., Di Clemente M., Russo G., "USV UHTC-based nose and WLE experimental test in Scirocco and CFD re building" Proceedings of 7th ESA Symposium on Aerothermodynamics for Space Vehicles, Bruges (Belgium), May 9th-12th , 2011.

[7] E. Trifoni, a. Del Vecchio, m. Di Clemente, v. De Simone, A. Martucci, C. Purpura, L. Savino, A. Cipullo, "Design of a Scientific Experiment on Expert Flap at Cira Scirocco Plasma Wind Tunnel", 7th European Symposium on Aerothermodynamics for Space Vehicles, Site Oud Sint-Jan, bruges, may 9-12, 2011.

[8] Acampora L., De Filippis F., Martucci A., Sorgi L., "3D Reconstruction of Thermal Images" Proceedings of 26th Aerospace Testing Seminar, Los Angeles (USA), March 2011. 